\title{
The Addition of Valproic Acid to Concurrent Radiation Therapy and Temozolomide Improves Patient Outcome: A Correlative Analysis of RTOG 0525, SEER and A Phase II NCI Trial
}

\author{
Andra V. Krauze*, Megan Mackey, Theresa Cooley-Zgela, Peter Mathen, Joanna H. Shih, Philip J. Tofilon, Lindsay Rowe, Mark \\ Gilbert, Kevin Camphausen
}

Radiation Oncology Branch, Center for Cancer Research, National Cancer Institute, NIH, 9000 Rockville Pike, Building 10, CRC Bethesda, MD 20892, USA

${ }^{\star}$ Corresponding author: Andra Krauze, Radiation Oncology Branch, Center for Cancer Research, National Cancer Institute, NIH, 9000 Rockville Pike, Building 10, CRC Bethesda, MD 20892, USA; Tel: (301) 496-5457; Fax: (301) 480-5437; E-mail: andra.krauze@bccancer.bc.ca

Received: December 12, 2019; Accepted: January 06, 2020; Published: January 11, 2020;

\begin{abstract}
Purpose/Objective(s): Valproic Acid (VPA) is an antiepileptic agent with HDACi (histone deacetylase inhibitor) activity shown to radiosensitize glioblastoma (GBM) cells. We evaluated the addition of VPA to standard radiation therapy (RT) and temozolomide (TMZ) in an open- label, phase II study (NCI-06-C-0112). The intent of the current study was to compare our patient outcomes with modern era standard of care data (RTOG 0525) and general population data (SEER 2006-2013).
\end{abstract}

Materials/Methods: 37 patients with newly diagnosed GBM were treated in a phase II NCI trial with daily VPA (25 mg/kg) in addition to concurrent RT and TMZ (2006 - 2013) and 411 patients with newly diagnosed GBM were treated in the standard TMZ dose arm of RTOG 0525 (2006 - 2008 ). Using the SEER database, adult patients (age > 15) with diagnostic codes 9440- 9443 (third edition (IDC-O-3) diagnosed between 2006 - 2013 were identified and 6083 were included in the analysis. Kaplan-Meier method was used to estimate OS and PFS. The effect of patient characteristics and clinical factors on OS and PFS was analyzed using univariate analysis and a Cox regression model. A landmark analysis was performed to correlate recurrence to OS and conditional probabilities of surviving an additional 12 months at diagnosis, 6, 12, 18, 24 and 30 months were calculated for both the trial data and the SEER data.

Results: Updated median OS in the NCI cohort was 30.9m (22.2- 65.6m), compared to RTOG $052518.9 \mathrm{~m}(16.8-20.3 \mathrm{~m})(\mathrm{p}=0.007)$ and the SEER cohort of $11 \mathrm{~m}$. Median PFS in the NCI cohort was $11.1 \mathrm{~m}(6.6-49.6 \mathrm{~m})$ compared to RTOG 0525 with a median PFS of 7.5m $(6.9-8.2 \mathrm{~m})(\mathrm{p}=0.004)$. Younger age, class V RPA and MGMT status were significant for PFS in both the NCI cohort and the RTOG 0525 cohort, in addition KPS was also significant for OS. In comparison to RTOG 0525, the population in the NCI cohort had a more favorable KPS and RPA, and a higher proportion of patients receiving bevacizumab after protocol therapy however with the exception of RPA (V) ( $8 \%$ vs $18 \%)(0.026)$, the effects of these factors on PFS and OS were not significantly different between the two cohorts.

Conclusion: Previously reported improvements in PFS and OS with the addition of VPA to concurrent RT and TMZ in the NCI phase II study were confirmed by comparison to both a trial population receiving standard of care (RTOG 0525) and a contemporary SEER cohort. These results provide further justification of a phase III trial of VPA/RT/TMZ.

\section{Introduction}

In the United States, primary brain tumors represent $2 \%$ of tumor subtypes with 23,000 new cases and 14,000 deaths per year with grade 4 glioblastoma (GBM) the most common. Standard therapy consists of maximal surgical resection followed by concurrent radiation therapy (RT) and temozolomide (TMZ) followed by adjuvant TMZ, which results in an overall survival (OS) of $27.2 \%$ at 2 years and $9.8 \%$ at 5 years [1]. Although, the efficacy of this therapy remains limited, attempts to increase the effectiveness of the RT/TMZ protocol $[2,3]$ have not been successful.
Since the pattern of recurrence following the RT/TMZ combination indicates failure in or adjacent to the initial RT treatment volume, enhancing the effectiveness of RT could lead to an improved therapeutic response. It is this premise that prompted the use of histone deacetylase inhibitors (HDACi) [4, 5]. Inhibition of HDAC activity has been shown to selectively increase tumor cell radiosensitivity in a variety of in vitro models and enhance radiationinduced growth delay of subcutaneous human tumor xenografts [4, 5]. HDACi reduces the repair of DNA double strand breaks (DSBs) leading to radiation-induced cell death $[4,5]$. 
Valproic acid (VPA), a non-enzyme-inducing antiepileptic drug (non-EI-AED), is orally bioavailable, crosses the blood-brain barrier and has minimal toxicity and thus, it provides an attractive option as a radiation sensitizer. In 2006 we initiated a study designed to investigate the safety, tolerability and effectiveness of concomitant RT/TMZ and relatively high dose VPA followed by adjuvant TMZ in patients with newly diagnosed GBM. The previously published median OS in the study was 29.6 months (range: 21-63.8 months) with a PFS of 10.5 months (range: 6.8-51.2 months) while toxicity was similar to that of other phase II studies involving RT/TMZ [6].

Over the past 10 years the reported median survival data for GBM has increased from 14 months to around 20 months [7]. The reasons for this increase are likely multifactorial including a combination of superior surgical resection, earlier initiation of systemic therapies at first recurrence and the management of patients within subspecialized neuro-oncology teams. Since our Phase II NCI trial did not include a standard of care arm, we compared the outcome of the patients treated on our Phase II trial with concurrent VPA with the outcomes of patients who received standard of care concurrent RT and TMZ in the modern era. Thus, we performed a secondary analysis of the RTOG 0525 standard of care arm in conjunction with our NCI data. The most recent molecular RPA based analysis of the RTOG 0525 standard arm included MGMT, Ki-67, mTOR and survivin in addition to age, KPS, extent of resection, and neurologic function, and revealed the median OS times for the three classes as 21.9, 16.6 and 9.4m [7]. Since both the $\mathrm{NCI}$ and the RTOG cohorts represent patients treated on trial who as a result tend to have superior outcomes, an additional comparison with data from GBM patients in the SEER database, who are more likely to be more representative of the GBM patient population at large, was also conducted. The intent was to assess the outcome of patients in the Phase II VPA trial in comparison with both modern era GBM patients treated on trial (RTOG 0525) and the population at large (SEER).

\section{Methods and Materials}

\section{Patient population}

\section{NCI Phase II study of VPA concurrent with chemoirradiation (CRT)}

The NCI study was a two center, open-label, phase II study (NCI-06-C-0112) that was conducted in patients with histologically confirmed GBM, aged 18 years or older and a life expectancy greater than 8 weeks, with surgery no more than 6 weeks prior to enrollment. Pathology review was obtained in all patients. Patients were required to have an ECOG performance status $\leq 2$ and adequate hematological, renal and hepatic function. Exclusion criteria included previous VPA, chemotherapy or radiotherapy treatment, a known disorder of urea metabolism and any history of a second malignancy other than nonmelanoma skin cancer or cervical cancer $<3$ years since diagnosis. The concurrent use of sulfamethoxazole, salicylates or naproxen was not allowed. The protocol was reviewed and approved by the NCI Institutional Review Board, and written informed consent was signed by all patients. 37 patients with newly diagnosed GBM were treated in a phase II NCI trial with daily VPA $(25 \mathrm{mg} / \mathrm{kg})$ in addition to concurrent RT and TMZ 2006 - 2013.

\section{RTOG 0525 population}

The RTOG 0525 study examined the effect of dose dense TMZ on 833 patients randomized to two arms (dose dense vs standard of care). Inclusion and exclusion criteria were largely similar to those of the phase II NCI study (patients older than age 18 years with a newly diagnosed, histologically confirmed GBM, KPS of at least 60 and adequate hematologic, renal, and hepatic function). Having verified that RTOG 0525 shares inclusion and exclusion criteria with our own study, we were specifically interested in the 411 patients who were randomized to and received standard of care in order to facilitate comparison with the phase II NCI study patient population. A secondary analysis request was submitted to NRG for the baseline characteristics (age, sex, KPS, type of surgical intervention, RPA class and MGMT status) of the patient population randomized to standard of care arm in RTOG0525. To further compare the impact of adjuvant treatment between these two populations, we requested the number of cycles of adjuvant TMZ the patient received and whether the patient did or did not receive Bevacizumab. In order to compare OS and PFS as the relevant endpoints we requested time to death or progression for the standard of care arm from initiation of treatment.

\section{SEER population}

Using the SEER database, adult patients (age > 15) with diagnostic codes 9440-9443 (third edition (IDC-O-3) diagnosed between 2006 - 2013 were identified and 6083 were included in the analysis. The intention was to analyze the GBM population captured in SEER over a similar time span as the patient populations captured in the NCI study and the RTOG 0525 studies.

\section{Statistical Analysis}

Pretreatment characteristics of the Phase II NCI study population and the RTOG 0525 population were compared using log rank test. Outcomes between the two studies were compared using log rank test and cox regression model for confounders. Kaplan-Meier method was used to estimate OS and PFS. The effect of patient characteristics and clinical factors on OS and PFS was analyzed using univariate analysis and a Cox regression model. A landmark analysis $[8,9]$ was performed to assess residual survival of additional 12 months.

\section{Results}

The NCI cohort represents 37 patients on the NCI phase II trial. Patient characteristics including the patient's performance status and RPA class as well as treatment details and progression and survival outcomes were available, however MGMT status was only available in $51 \%$ of the patients as many patients were accrued prior to standard MGMT testing (Table 1). The RTOG 0525 cohort represents 411 patients included on the standard of care arm of RTOG 0525. Similar to the NCI cohort, patient's characteristics are available and MGMT status was available in $91 \%$ of the patients in this cohort (Table 1). In comparison to RTOG 0525, the population in the NCI cohort had a more favorable KPS and RPA, and a higher proportion of patients receiving bevacizumab as salvage (Table1). However, with the exception of RPA (V) (8\% vs 18\%), the effects of these factors on PFS 
and OS were not significantly different between the two cohorts (Table 2 and Table 3). Median OS in the NCI cohort was $30.9 \mathrm{~m}(22.2-65.6 \mathrm{~m})$, compared to RTOG $052518.9 \mathrm{~m}$ (16.8- 20.3) ( $\mathrm{p}=0.003)$ (Figure 1), and SEER cohort $11 \mathrm{~m}$ (supplemental table). Younger age, KPS and class V RPA, were significant for OS in both the NCI cohort and the RTOG 0525 cohort while MGMT status was only significant for the RTOG 0525 cohort (Table 2). Median PFS was 11.1 and $7.5 \mathrm{~m}$ in the NCI study and the RTOG study respectively (Figure 2). PFS was superior in the NCI study as compared to the RTOG study at 6, 12 and 24 months $(\mathrm{p}=0.0005)$ (Figure 2). Younger age, class V RPA and MGMT status were significant for PFS in both the NCI cohort and the RTOG 0525 cohort (Table 3). The SEER cohort represents a population of patients captured in the US SEER database. Unlike the NCI or RTOG cohorts, patient's performance status, RPA class and MGMT status as well as the extent of systemic management and PFS were not recorded within the database and therefore were not available for comparison.

Since significant factors that underlie the ability to analyze survival are not available across the all three data sets, a landmark analysis was used to estimate the survival probabilities in each of the data sets conditional on the patients having survived at least 6 months. From the analysis, with a landmark time of $6 \mathrm{~m}$, the percent of patients surviving an additional $12 \mathrm{~m}$ was 77,57 and 43 in the NCI study, RTOG 0525 and SEER respectively (Table 4 ). The difference was statistically significant at the initiation of treatment, at $6 \mathrm{~m}$ and at $18 \mathrm{~m}$. VPA patients' survival was superior to both RTOG and SEER patients up until $30 \mathrm{~m}$ when they coalesced to a similar likelihood of surviving an additional $12 \mathrm{~m}$ (Figure 3 ).

Table 1. Pretreatment characteristics. Recursive partitioning analysis (RPA), gross total resection(GTR), sub-total resection (STR), valproic acid (VPA), methylguanine-DNA methyltransferase (MGMT), epidermal growth factor receptor (EGFR)

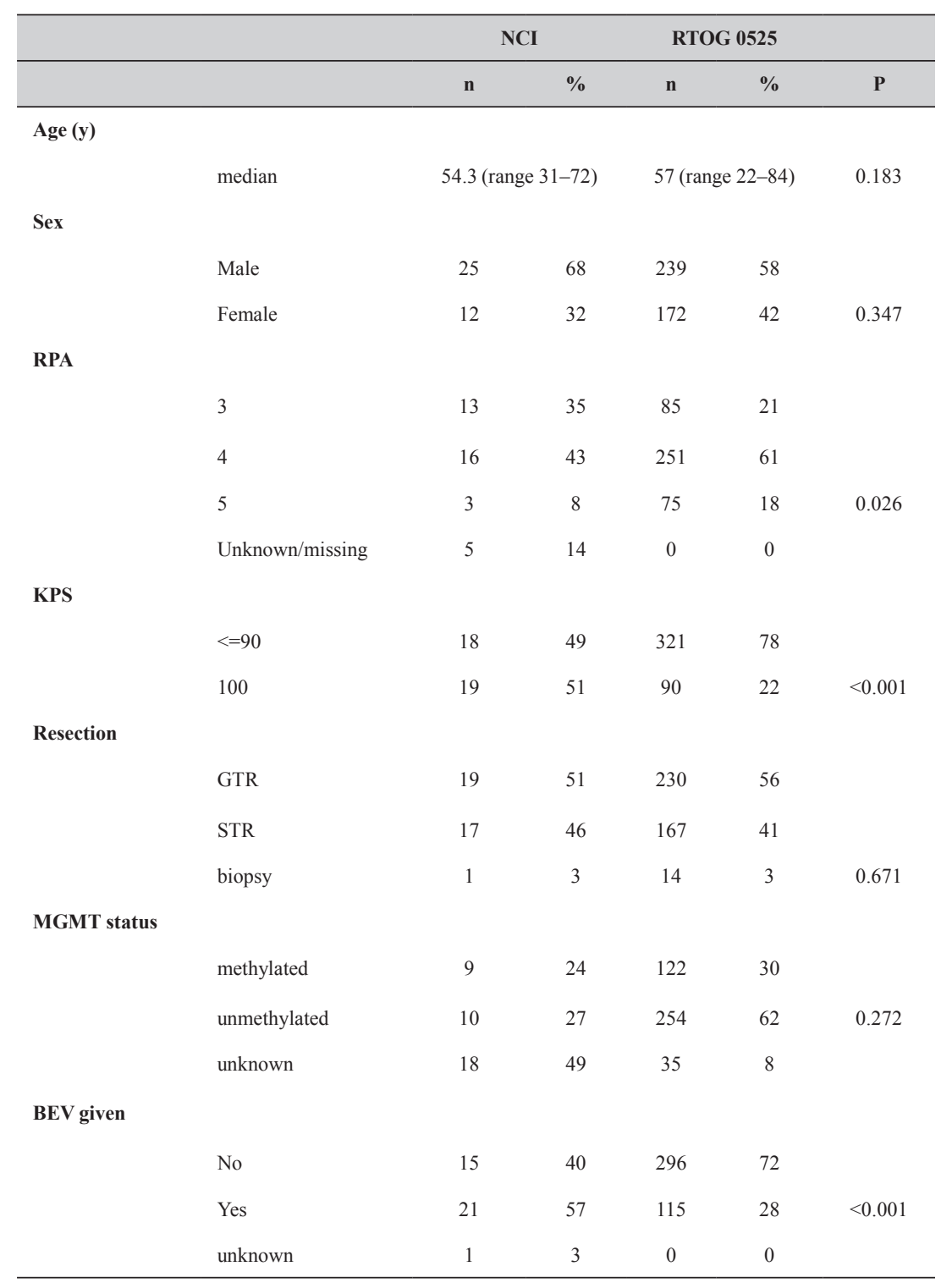


Table 2. Univariate analysis for OS.

\begin{tabular}{|c|c|c|c|c|c|c|c|}
\hline & NCI & & & RTOG 0525 & & & Difference in $\mathrm{HR}$ \\
\hline & HR & $95 \%$ CI & p-value & HR & $95 \% \mathrm{CI}$ & p-value & P-value \\
\hline Age (continuous variable)* & 1.6 & $1.0-2.4$ & 0.046 & 1.3 & $1.2-1.4$ & $<0.001$ & 0.399 \\
\hline Age: $50-60$ years & 2.2 & $0.8-6.1$ & 0.147 & 1.6 & $1.2-2.1$ & 0.002 & 0.564 \\
\hline Age: $>60$ years & 3.2 & $1.0-10.0$ & 0.043 & 1.8 & $1.4-2.4$ & $<0.001$ & 0.331 \\
\hline KPS $(<=90)$ & 2.7 & $1.1-6.4$ & 0.029 & 1.5 & $1.1-1.9$ & 0.007 & 0.205 \\
\hline RPA (class IV) & 1.8 & $0.6-5.0$ & 0.266 & 1.5 & $1.1-2.1$ & 0.004 & 0.773 \\
\hline RPA (class V) & 5.7 & $1.3-26.1$ & 0.024 & 2.2 & $1.5-3.2$ & $<0.001$ & 0.231 \\
\hline Extent of resection $=\mathrm{STR}$ & 1.4 & $0.6-3.1$ & 0.383 & 1.1 & $0.9-1.4$ & 0.448 & 0.53 \\
\hline Avastin (yes) & 0.7 & $0.3-1.6$ & 0.389 & 0.7 & $0.5-0.9$ & 0.002 & 1.00 \\
\hline MGMT (unmethylated) & 3.6 & $0.9-13.9$ & 0.068 & 1.9 & $1.5-2.5$ & $<0.001$ & 0.390 \\
\hline
\end{tabular}

${ }^{*} \mathrm{HR}$ corresponds to increase of age by 10 years

Table 3. Univariate analysis for PFS

\begin{tabular}{|c|c|c|c|c|c|c|c|}
\hline & & NCI & & RTOG 0525 & & & Difference in $\mathrm{HR}$ \\
\hline & HR & $95 \% \mathrm{CI}$ & p-value & HR & $95 \% \mathrm{CI}$ & p-value & p-value \\
\hline Age (continuous variable)* & 1.3 & $0.9-2.0$ & 0.156 & 1.2 & $1.1-1.3$ & $<0.001$ & 0.59 \\
\hline Age: $50-60$ years & 1.6 & $0.6-4.2$ & 0.297 & 1.4 & $1.1-1.8$ & 0.008 & 0.775 \\
\hline Age: $>60$ years & 2.9 & $1.0-8.1$ & 0.048 & 1.5 & $1.2-1.9$ & 0.002 & 0.231 \\
\hline KPS $(<=90)$ & 1.8 & $0.8-3.8$ & 0.128 & 1.1 & $0.8-1.4$ & 0.517 & 0.21 \\
\hline RPA (class IV) & 1.4 & $0.5-3.6$ & 0.517 & 1.2 & $0.9-1.6$ & 0.128 & 0.829 \\
\hline RPA (class V) & 12.2 & $2.4-62.9$ & 0.003 & 1.7 & $1.2-2.4$ & 0.002 & 0.021 \\
\hline Extent of resection $=S T R$ & 1.1 & $0.5-2.4$ & 0.743 & 1.1 & $0.9-1.3$ & 0.482 & 0.90 \\
\hline Avastin (yes) & 1.4 & $0.6-3.1$ & 0.462 & 1.3 & $1.0-1.6$ & 0.029 & 0.89 \\
\hline MGMT (unmethylated) & 3.9 & $1.0-14.6$ & 0.045 & 1.6 & $1.2-2.0$ & $<0.001$ & 0.187 \\
\hline
\end{tabular}

*HR corresponds to increase of age by 10 years

\section{Discussion}

In recent years the published outcomes of GBM patients on trial has improved as compared to published Stupp data $[1,7]$, nonetheless, outcomes for GBM patients remain poor. Between 2006 and 2013 we carried out a Phase II trial with the goal of investigating the safety, tolerability and effectiveness of concomitant RT/TMZ and relatively high dose of VPA followed by adjuvant TMZ in patients with newly diagnosed GBM [6]. In order to compare the outcome of our Phase II NCI study with modern GBM treatment and outcomes, we performed a secondary analysis of the standard of care arm of RTOG 0525. To ensure adequate comparison with the GBM population at large, we also carried out a SEER analysis spanning the same time period as the previous two trials (2006-2013). The NCI study and RTOG study patients were sufficiently similar to allow for a meaningful analysis although the NCI patients had superior KPS, RPA and use of Bevacizumab. None of these factors were found to have a statistically significant impact on the analysis. We found that the NCI study patients had a superior PFS and OS compared to either the RTOG 0525 patients or the SEER patients, who in turn had the poorest survival.

Median OS for the NCI patients far surpassed RTOG 0525 and the SEER cohort outcomes at $30.9 \mathrm{~m}$ (22.2-65.6). It also surpassed other modern published GBM data $[2,3,7]$. The effect observed on OS was sustained to 30 months post initiation of treatment. In recent years, landmark analyses have been employed in order to contextualize outcome results by clarifying the relationship between progression 
and survival $[8,9]$. We carried out a landmark analysis on the premise that those patients that had not died at the 12 month time point may have superior biological behavior possibly distinguished by biomarkers such as MGMT, Ki-67, survivin or mpTOR as well as other as yet undiscovered biomarkers which may correlate with the administration of VPA. The analysis suggests a significant benefit to the administration of VPA with $71 \%$ of patients having a probability of surviving another 12 months at the $18 \mathrm{~m}$ time point in the NCI study as compared to $51 \%$ in the RTOG 0525 and $48 \%$ in the SEER cohort. The data also showcases that fact that the modern day RTOG 0525 cohort, presumably representing a highly selected, superior performing population nonetheless has probability of surviving another 12 months that is very similar to the SEER cohort once they pass the $6 \mathrm{~m}$ time point. By contrast the NCI VPA treated cohort approaches similar probabilities of survival to the SEER cohort much later, approaching 30 months. This could indicate a potential alteration in tumor biology that occurs in patients treated with VPA which could be explored further in larger cohorts.

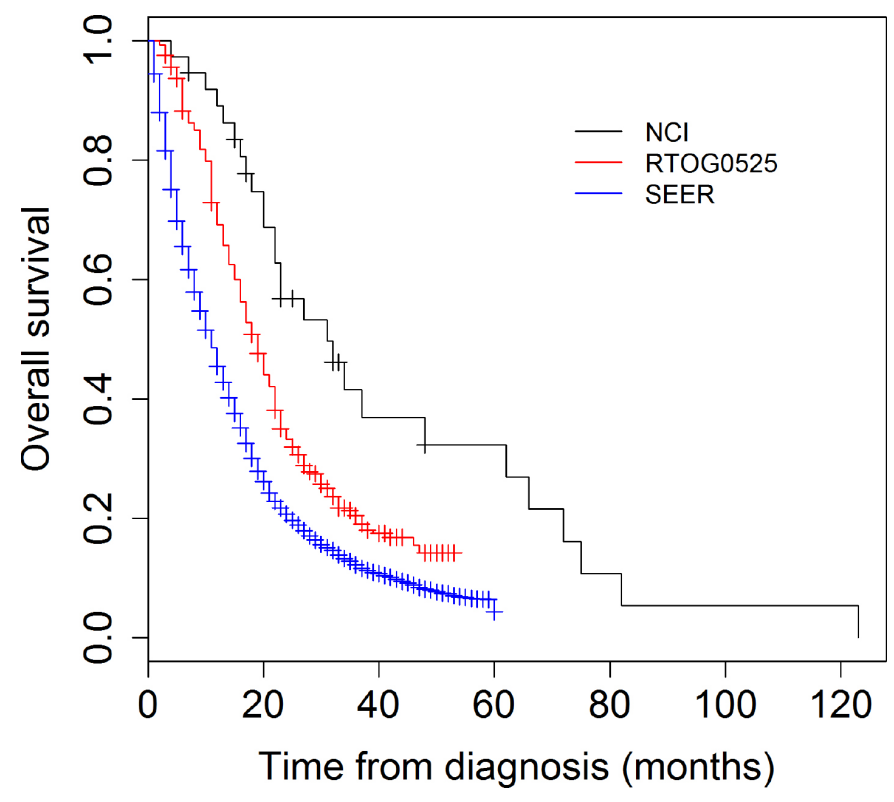

Figure 1. Overall survival (OS) comparison NCI phase II study, RTOG 0525 and SEER 2006-2013.

In our phase II study we explored the effectiveness of VPA as a radiosensitizer [6] and the hypothesis that VPA favorably alters tumors response continues to be explored in the literature [10-18]. While a recent analysis of four contemporary randomized clinical trials [14] showed no improvement in outcome to the addition of VPA, dose dependency was not addressed in this analysis and existing data suggests that the effect of VPA is dose dependent [15]. By contrast a 2014 meta-analysis suggested improved survival of patients with VPA administration $[16,17]$ although dose response was not addressed. It is possible that the effects of VPA may include modification of tumor behavior that may be both disease and dose dependent. Biomarker studies aimed at exploring possible avenues underlying the effects of VPA are ongoing within the NCI study cohort and in the literature at large [18].

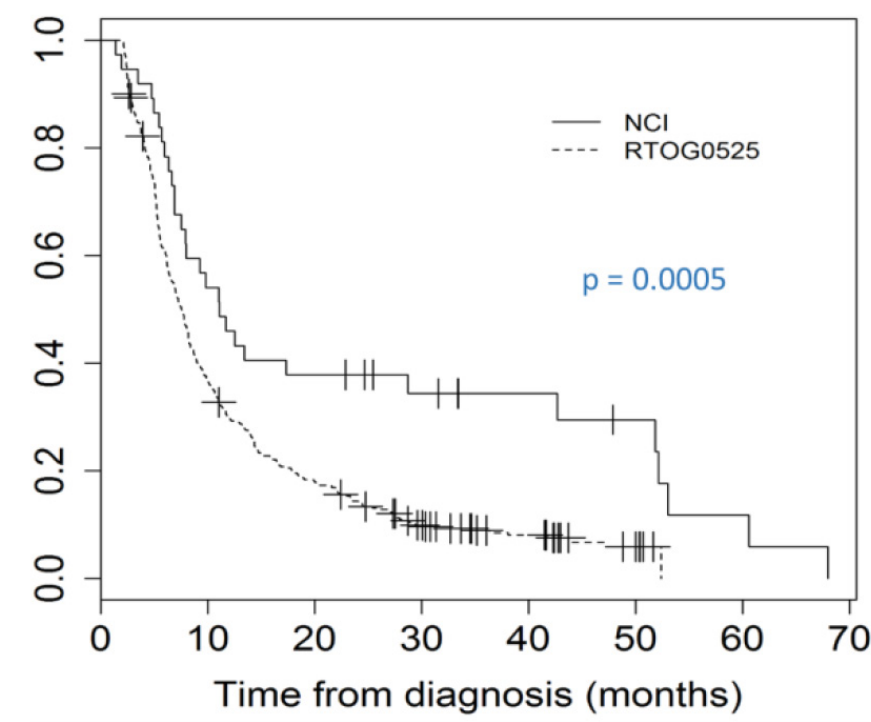

Figure 2. Kaplan-Meier analysis of progression free survival (PFS) between RTOG 0525 and the phase II NCI study examining the addition of Valproic acid (VPA) to concurrent radiation (RT) and temozolomide (TMZ).

There are several limitations to our analysis, including the fact that the number of patients on the standard of care arm in RTOG 0525 (411) greatly exceeded the number of patients in the NCI study (37), and that MGMT status was unknown in $49 \%$ of NCI study patients, as the bulk of the study was carried out preceding the era of wide spread MGMT methylation testing. In the phase II NCI study time to death or progression was calculated from the initiation of VPA which preceded the initiation of RT and TMZ by 1 week, while in RTOG 0525 time to death or progression was calculated from initiation of concurrent CRT and it was not possible to account for this discrepancy, although the difference of 1 week is unlikely to significantly alter our conclusions. In addition, progression on RTOG 0525 was determined according to Macdonald criteria, whereas the NCI study used RECIST criteria, although both studies accounted for pseudoprogression in determining response. However since SEER does not capture patient characteristics such as KPS, RPA, progression data or MGMT status, a comparison of these characteristics between the NCI study and RTOG 0525 and SEER was not possible. We understand that while anticonvulsant (AED) usage was collected on RTOG 0525, the use of VPA specifically, as a type of AED was not collected.

\section{Conclusion}

Previously reported improvements in PFS and OS with the addition of VPA to concurrent RT and TMZ in the NCI phase II study were confirmed in comparison to both a trial population receiving standard of care (RTOG 0525) and a contemporary SEER cohort. These results warrant further consideration of VPA for analysis in a phase III trial.

\section{Declarations}

\section{Ethics approval and consent to participate}

This study reports on data collected from humans. This research was exempt from NCI IRB approval as one of the six exemptions 
to the regulatory requirements are described in section 46.101(b) of 45 CFR 46, subsection (4): Research involving the collection or study of existing data, documents, records, pathological specimens, or diagnostic specimens, if these sources are publicly available or if the information is recorded by the investigator in such a manner that subjects cannot be identified, directly or through identifiers linked to the subjects.

Table 4. Landmark analysis. Probability and $95 \% \mathrm{CI}$ of surviving additional 12 months.

\begin{tabular}{|c|c|c|c|c|c|c|c|c|c|c|c|}
\hline & NCI & & & RTOG & & & & SEER & & & \\
\hline $\begin{array}{l}\text { Time from } \\
\text { initiation of } \\
\text { treatment } \\
\text { (months) }\end{array}$ & $\begin{array}{c}\text { Probability } \\
\text { of surviving } \\
\text { additional } \\
12 \text { months }\end{array}$ & $\begin{array}{c}\text { Lower } \\
95 \% \text { CI }\end{array}$ & $\begin{array}{c}\text { Upper } \\
95 \% \text { CI }\end{array}$ & $\begin{array}{l}\text { Probability } \\
\text { of surviving } \\
\text { additional } \\
12 \text { months }\end{array}$ & $\begin{array}{c}\text { Lower } \\
95 \% \text { CI }\end{array}$ & $\begin{array}{c}\text { Upper } \\
95 \% \text { CI }\end{array}$ & $\mathbf{P}$ & $\begin{array}{c}\text { Probability } \\
\text { of surviving } \\
\text { additional } \\
12 \text { months }\end{array}$ & $\begin{array}{c}\text { Lower } \\
95 \% \\
\text { CI }\end{array}$ & $\begin{array}{c}\text { Upper } \\
95 \% \\
\text { CI }\end{array}$ & P-value* \\
\hline 0 & 0.92 & 0.83 & 1 & 0.70 & 0.66 & 0.75 & $<0.001$ & 0.45 & 0.44 & 0.47 & 0.000 \\
\hline 12 & 0.61 & 0.46 & 0.81 & 0.49 & 0.43 & 0.55 & 0.167 & 0.43 & 0.41 & 0.44 & 0.032 \\
\hline 18 & 0.71 & 0.55 & 0.92 & 0.51 & 0.45 & 0.59 & 0.049 & 0.48 & 0.46 & 0.51 & 0.016 \\
\hline 24 & 0.73 & 0.53 & 1 & 0.62 & 0.53 & 0.72 & 0.385 & 0.56 & 0.53 & 0.59 & 0.165 \\
\hline 30 & 0.69 & 0.47 & 1 & 0.61 & 0.5 & 0.75 & 0.610 & 0.62 & 0.58 & 0.65 & 0.587 \\
\hline
\end{tabular}

*Comparing survival probability between $\mathrm{NCI}$ and SEER

^RTOG 0525

- VPA data from diagnosis

$\rightarrow$ SEER 2006-2013

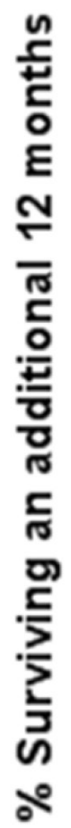

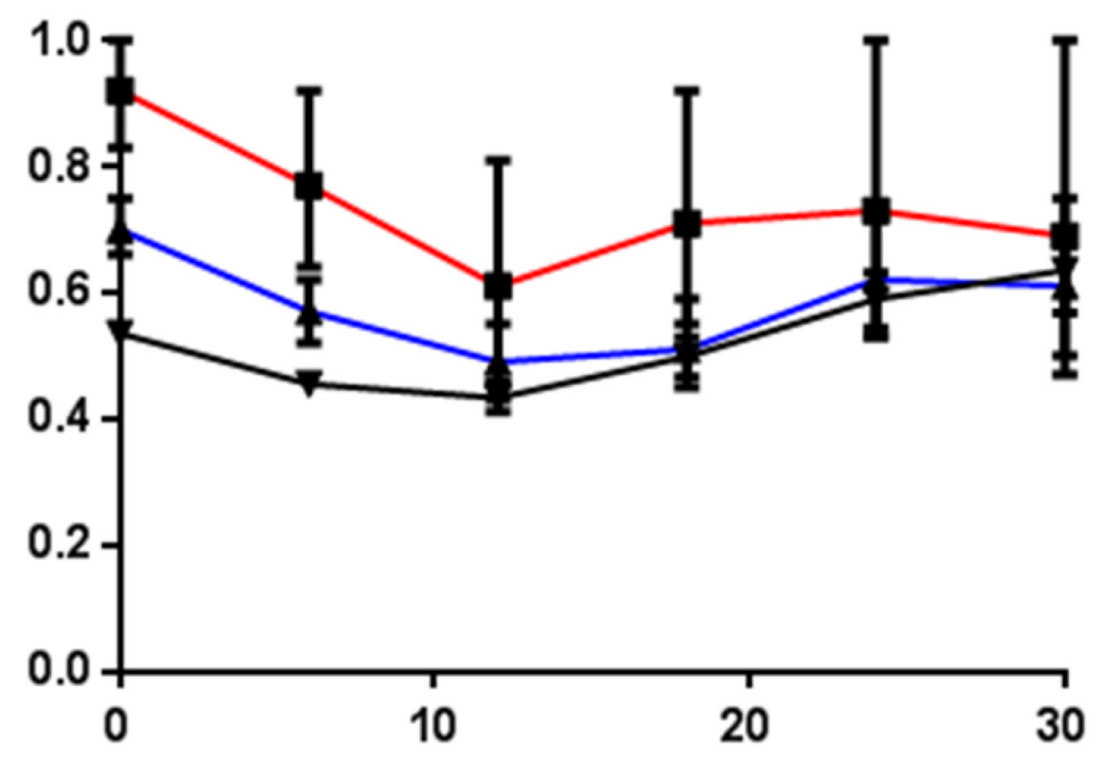

Time (months)

Figure 3. Landmark analysis - Kaplan-Meier survival estimate vs. timing of recurrence using landmark analysis setting the landmark time at 6 months. 
Andra Krauze (2020) The Addition of Valproic Acid to Concurrent Radiation Therapy and Temozolomide Improves Patient Outcome:

A Correlative Analysis of RTOG 0525, SEER and A Phase II NCI Trial

Supplemental Table. Comparison of PFS and OS between NCI and RTOG 0525 study cohort. Log-rank test was employed to compare PFS and OS between NCI and RTOG 0525 study cohort and between the NCI and SEER cohort.

\begin{tabular}{|c|c|c|c|c|c|}
\hline & \multicolumn{2}{|c|}{$\mathrm{NCI}(\mathrm{n}=37)$} & \multicolumn{2}{|c|}{ RTOG $0525(n=411)$} & \multirow{2}{*}{$\begin{array}{c}\begin{array}{c}\text { SEER GBM 2006-2013 } \\
(\mathbf{n}=6083)\end{array} \\
\text { OS }\end{array}$} \\
\hline & PFS & OS & PFS & OS & \\
\hline \# of events (\%) & $30(81)$ & $27(73)$ & $374(91)$ & $320(78)$ & $5359(88)$ \\
\hline $\begin{array}{l}\text { median } \\
\text { (months) }(95 \% \mathrm{CI})\end{array}$ & $11.1(8.0,51.9)$ & $30.9(22.2,65.6)$ & $7.5(6.9,8.2)$ & $18.9(16.8,20.3)$ & 11 \\
\hline 6 months (\%) & $78.4(66.2,92.8)$ & $97.3(92.2,100)$ & $61(56.5,65.9)$ & $91.9(89.3,94.6)$ & $65.5(64.3,66.7)$ \\
\hline 12 months (\%) & $45.9(32.4,65.2)$ & $91.7(83.2,100)$ & $30(25.9,34.8)$ & $70.4(66.1,75)$ & $45.5(44.2,46.7)$ \\
\hline 24 months (\%) & $37.8(25,57.2)$ & $56.4(41.9,75.8)$ & $14.4(11.3,18.2)$ & $34.2(29.9,39.2)$ & $20.7(19.6,21.8)$ \\
\hline p-value ${ }^{*}$ & & & $0.0005^{*}$ & $0.003^{*}$ & $<0.001^{\star *}$ \\
\hline
\end{tabular}

*Log-rank test comparing PFS and OS between NCI and RTOG0525 study cohort; **log-rank test comparing

OS between NCI and SEER cohort

\section{Availability of data and material}

The datasets supporting the conclusions of this article are included within the article and its additional files.

\section{Funding}

This work was supported in part by the Centers for Cancer Research, NCI. \#ZIASC 010372.

\section{Authors' contributions}

AVK conceived the study, participated in its design and coordination, collected patient data, performed statistical data analysis and drafted the manuscript.

MSD, CMG, DJH, SS, RL treated patients included in the original phase II study and RTOG 0525 (MG) and collected patient data.

JS performed the statistical data analysis.

$\mathrm{KC}, \mathrm{PJT}$ conceived the original NCI phase II study.

$\mathrm{KC}$ and AVK conceived the secondary analysis and participated in draft of the manuscript.

\section{Acknowledgements}

This work was supported in part by the intramural program of the NCI under funding ZIA-SC- 010373.

\section{List of Abbreviations}

CRT - Concurrent Chemoirradiation CNS - Central Nervous System EORTC - European Organisation for Research and Treatment of Cancer GBM - Glioblastoma

KPS - Karnofsky Performance Status MRI - Magnetic Resonance Imaging NCI - National Cancer Institute

OS - Overall Survival

PFS - Progression Free Survival
RPA - Recursive Partitioning Analysis RT - Radiation Therapy TMZ - Temozolomide

\section{References}

1. Stupp R, Hegi ME, Mason WP, van den Bent MJ, Taphoorn MJ, et al. (2009) European Organisation for Research and Treatment of Cancer Brain Tumour and Radiation Oncology Groups; National Cancer Institute of Canada Clinical Trials Group.Effects of radiotherapy with concomitant and adjuvant temozolomide versus radiotherapy alone on survival in glioblastoma in a randomised phase III study: 5-year analysis of the EORTC-NCIC trial. Lancet Oncol 10: 459-466.

2. Gilbert MR, Dignam JJ, Armstrong TS, Terri S. Armstrong, Jeffrey S. Wefel, Deborah T. Blumenthal, et al. (2014) A randomized trial of bevacizumab for newly diagnosed glioblastoma. $N$ Engl J Med 370: 699-708.

3. Chinot OL, Wick W, Mason W, Roger Henriksson, Frank Saran, et al. (2014) Bevacizumab plus radiotherapy-temozolomide for newly diagnosed glioblastoma. N Engl J Med 370: 709-722.

4. Camphausen K, Cerna D, Scott T, Sproull M, Burgan WE, et al. (2005) Enhancement of in vitro and in vivo tumor cell radiosensitivity by VPA. Int $J$ Cancer 114: 380 386

5. Camphausen K, Scott T, Sproull M (2004) Enhancement of xenograft tumor radiosensitivity by the histone deacetylase inhibitor MS-275 and correlation with histone hyperacetylation. Clin Cancer Res 10: 6066-6071.

6. Krauze AV, Myrehaug SD, Chang MG, Holdford DJ, Smith S, Shih J, et al. (2015) A Phase 2 Study of Concurrent Radiation Therapy, Temozolomide, and the Histone Deacetylase Inhibitor Valproic Acid for Patients With Glioblastoma. Int J Radiat Oncol Biol Phys 92: 986-992.

7. Erica Hlavin Bell, Stephanie L. Pugh, Joseph P. McElroy, Mark R. Gilbert, Minesh Mehta, et al. (2018) Molecular- Based Recursive Partitioning Analysis (RPA) Model for Glioblastoma in the Temozolomide Era: A Correlative Analysis Based Upon NRG Oncology RTOG 0525. JAMA Oncol Author manuscript; available in PMC 2018 Jun 1.

8. Prados M, Cloughesy T, Samant M, Fang L, Wen PY, et al. (2011) Response as a predictor of survival in patients with recurrent glioblastoma treated with bevacizumab. Neuro Oncol 13: 143-151.

9. Polley MY, Lamborn KR, Chang SM, Butowski N, Clarke JL, et al. (2010) Sixmonth progression-free survival as an alternative primary efficacy endpoint to overall survival in newly diagnosed glioblastoma patients receiving temozolomide. Neuro Oncol 12: 274-282.

10. Ochiai S, Nomoto Y, Yamashita Y, Watanabe Y, Toyomasu Y, et al. (2016) Roles of Valproic Acid in Improving Radiation Therapy for Glioblastoma: a Review of Literature Focusing on Clinical Evidence. Asian Pac J Cancer Prev 17: 463-466.

11. Redjal N, Reinshagen C, Le A, Walcott BP, McDonnell E, et al. (2016) Valproic acid, compared to other antiepileptic drugs, is associated with improved overall and progression- free survival in glioblastoma but worse outcome in grade II/III gliomas treated with temozolomide. J Neurooncol 127: 505-514. 
Andra Krauze (2020) The Addition of Valproic Acid to Concurrent Radiation Therapy and Temozolomide Improves Patient Outcome: A Correlative Analysis of RTOG 0525, SEER and A Phase II NCI Trial

12. Eckert M, Klumpp L, Huber SM (2017) Cellular Effects of the Antiepileptic Drug Valproic Acid in Glioblastoma. Cell Physiol Biochem 44: 1591-1605.

13. Thotala D, Karvas RM, Engelbach JA, Garbow JR, Hallahan AN, et al. (2015) Valproic acid enhances the efficacy of radiation therapy by protecting normal hippocampal neurons and sensitizing malignant glioblastoma cells. Oncotarget 6: 35004-35022.

14. Happold C, Gorlia T, Chinot O, Gilbert MR, Nabors LB, et al. (2016) Does Valproic Acid or Levetiracetam Improve Survival in Glioblastoma? A Pooled Analysis of Prospective Clinical Trials in Newly Diagnosed Glioblastoma. J Clin Oncol 34: 731-739.

15. Riva G, Butta V, Cilibrasi C, Baronchelli S, Redaelli S, et al. (2016) Epigenetic targeting of glioma stem cells: Short-term and long-term treatments with valproic acid modulate DNA methylation and differentiation behavior, but not temozolomide sensitivity. Oncol Rep 35: 2811-2824.
16. Yuan Y, Xiang W, Qing M, Yanhui L, Jiewen L, et al (2014) Survival analysis for valproic acid use in adult glioblastoma multiforme: a meta-analysis of individual patient data and a systematic review. Seizure 23: 830-835.

17. Lu VM, Texakalidis P, McDonald KL, Mekary RA, Smith TR (2018) The survival effect of valproic acid in glioblastoma and its current trend: a systematic review and meta-analysis. Clin Neurol Neurosurg 174: 149-155.

18. Adam Louis Cohen, Leigh Neumayer, Rachel Factor, Kenneth Boucher, Mark L. Wade, et al. (2016) A phase 1 window of opportunity study of valproic acid (VPA) in breast cancer testing a gene expression biomarker. Journal of Clinical Oncology 34: $1084-1084$

Citation:

Andra Krauze, Megan Mackey, Theresa Cooley-Zgela, Peter Mathen, Shih JH, et al. (2020) The Addition of Valproic Acid to Concurrent Radiation Therapy and Temozolomide Improves Patient Outcome: A Correlative Analysis of RTOG 0525, SEER and A Phase II NCI Trial. Cancer Stud Ther J Volume 5(1): 1-8. 